\title{
Psychiatry in the new undergraduate curriculum
}

\section{Howard Ring, David Mumford \& Cornelius Katona}

Recognising the vast extent of psychiatric morbidity internationally and the burden of mental illness on people, communities and nations, the World Psychiatric Association and the World Federation for Medical Education have recently published global guidelines for developing core curricula in psychiatry for medical students (Walton \& Gelder 1999). More locally, major changes are taking place in undergraduate medical education throughout the UK. These changes represent a response to the appreciation, both by medical schools and by the General Medical Council (GMC), of two major pressures in undergraduate education. The first is that students have been asked over the years to accumulate more and more factual knowledge while the knowledge base in medicine itself expands and changes more rapidly. The second is that both understanding of illness and delivery of care are developing an increasing focus on the role of the community and community support. These general pressures have led to a number of specific recommendations, initially put forward by the GMC in their document Tomorrow's Doctors (GMC,1993). This document encourages the reduction of 'core knowledge' taught to medical students to $65 \%$ of what has previously been taught, together with the identification of special study modules (SSMs), which would fill the remaining time in the curriculum. These SSMs would allow students to explore areas of particular interest in greater depth than was previously possible.

In constructing a core curriculum in undergraduate psychiatry, course organisers should acknowledge the guidance from the GMC that core knowledge be reduced by $35 \%$. Within psychiatry, as within other subject areas, such an exercise entails a degree of selectivity with respect to what is retained and what is left out. In order to pursue in a rational way the process of identifying core material, it is appropriate to consider what the aims of the teaching should be. It was the view of the GMC (1993) that the core curricula should be focused in such a way that graduates would qualify with the appropriate knowledge, skills and attitudes to enable them to perform appropriately during their pre-registration house officer attachments. Although such an aim is clearly important, the majority of those practising as doctors will not undertake any postgraduate training in psychiatry - yet in most medical specialities, patients may have some degree of psychiatric symptomatology. Hence, as well as equipping a newly qualified doctor for the first year of clinical practice, some thought should be given to ensuring that the psychiatry taught in the undergraduate curriculum will be appropriate to provide an adequate basis for a full medical career in any of a large number of specialities. At its simplest, such an aim may be met by ensuring that students know how to take a competent psychiatric history and perform a mental state examination. However, attaining these core skills will be facilitated by a knowledge of specific psychiatric symptoms and how they come together in various conditions.

The need to review the curriculum in psychiatry applies to all schools. Similarly, methods of teaching are being widely re-examined. This process is driven both by the increasing pressure on time across the undergraduate course and by a wish to develop teaching methods that encourage greater student participation and deeper learning. Although some of the demands for curriculum modernisation come from central bodies, such as the GMC and the

Howard Ring is Senior Lecturer in Psychiatry and Director of the undergraduate Brain and Behaviour program at St Bartholomew's and the Royal London School of Medicine (Whitechapel Road, London E1 1BB). David Mumford is Senior Lecturer in Psychiatry and Director of Medical Education at the University of Bristol. He has been involved in curriculum reform since 1995. Cornelius Katona is Foundation Professor of Psychiatry of the Elderly at the Royal Free and University College Medical School, and Dean of the Royal College of Psychiatrists. 
Higher Education Funding Councils, individual schools and universities retain control of the ways in which these demands are met. The consequence of this relative autonomy is that across the country, teaching methods and content are being reexamined. In some cases, this is likely to lead to individual schools 'reinventing the wheel'. One way to minimise such duplication of effort, and at the same time facilitate the sharing of best practice, is for individual schools to be aware of the work that is being done by the others. This paper presents results from a survey of the views of undergraduate psychiatry course organisers as to what in their school constitutes a 'core curriculum' in psychiatry and what teaching methods are currently employed in the psychiatric education of medical students.

\section{Survey of psychiatric teaching}

Each medical school in the UK and Ireland was sent a questionnaire exploring current local views on the teaching of psychiatry. These were completed by the individual psychiatrist within each school who held responsibility for teaching psychiatry. Responses to these questionnaires were obtained from 19 medical schools in the UK and four medical schools in the Republic of Ireland.

\section{What constitutes a core curriculum in psychiatry?}

The Education Committee of the Royal College of Psychiatrists previously published a preliminary document entitled Core Psychiatry for Tomorrow's Doctors (Working Party of the Education Committee of the Royal College of Psychiatrists, 1997). The current survey sought opinions on the content of that document.

The conditions believed by the great majority of respondents $(>85 \%)$ of the survey to represent core conditions in the undergraduate psychiatry curriculum are listed in Box 1. All respondents agreed that for these conditions, students should at least be familiar with information describing prevalence, presentation, aetiology and principles of management.

The majority $(>50 \%)$ of respondents also agreed that a further number of conditions (listed in Box 2) should be considered as core conditions in the undergraduate psychiatry curriculum - although taught in less depth than the conditions listed in Box 1, with the focus largely on presentation and broad principles of management.

\section{Box 1. Conditions thought by $>85 \%$ of respondents to represent core conditions in the undergraduate psychiatry curriculum \\ Acute confusional states and dementias \\ Affective disorders \\ Anxiety, panic and phobias \\ Conduct and emotional disorders of child- hood and adolescence \\ Obsessive-compulsive disorder \\ Schizophrenia \\ Substance misuse and dependence}

All respondents agreed that an understanding of relevant Mental Health Act material was important. With respect to therapeutics, it was agreed that knowledge of antidepressants, anxiolytics, hypnotics, antipsychotics, mood stabilisers and electroconvulsive therapy should be included. It was agreed by 19 out of the 23 respondent schools (83\%) that the principles of different forms of psychotherapy, particularly supportive psychotherapy, psychoanalytic psychotherapy and cognitive-behavioural approaches, should be taught. However, only $70 \%$ of schools considered that the psychiatry of learning disability represented a core subject.

The core skills agreed to be important are listed in Box 3. In addition, 21 out of the 23 respondents (91\%) considered that some understanding of risk assessment with respect to patients' potential to harm others was also appropriate for inclusion in the undergraduate curriculum. It was also reported that this latter topic should be dealt with only in a relatively general fashion and that the role of expert opinions should be emphasised.

Considering the inclusion of 'attitudes' within the curriculum, respondents noted these may be

Box 2. Additional conditions thought by $>\mathbf{5 0} \%$ of respondents to represent core conditions in the undergraduate psychiatry curriculum

Adjustment reactions

Eating disorders

Normal and abnormal grief

Personality disorders

Pervasive developmental disorders

Post-traumatic stress disorder

Psychological problems complicating physical illness

Psychosexual disorders 


\section{Box 3. Core skills in psychiatry}

Ability to communicate effectively with mentally ill patients of all ages and developmental levels

Ability to take a full psychiatric history and mental state examination

Ability to formulate a differential diagnosis

Ability to consider family relationships and their impact on individuals

Ability to perform physical and simple psychological investigations in patients presenting with psychiatric symptoms

Ability to assess suicidal risk

difficult to teach and to assess. Nevertheless, it was agreed by $90 \%$ that students should, during their attachment to psychiatry, develop and demonstrate an empathic understanding of the emotional problems of patients of all ages and developmental levels. In addition, there was similar agreement that it was appropriate for students to demonstrate a commitment to maximising the social integration of patients with mental health problems and to be sensitive to patient concerns about the stigma attaching to mental illness.

\section{Teaching methods}

Another of the aims of undergraduate education in medicine identified by the GMC (1993) was that during their study, students should develop appropriate attitudes to the acquisition and employment of knowledge to enable them to function well from the time they qualify and to be able to pursue appropriate continuing professional development with the appropriate skills for lifelong learning. Hence, having identified a core curriculum, schools must develop a structure for teaching that will satisfy these aims. Such a structure requires consideration of issues such as:

- Where, within the five or six years of the undergraduate curriculum, should core psychiatry teaching be offered?

- How should psychiatry be integrated with other subjects?

- What teaching methods and assessments could be employed?

- Who should actually deliver the teaching?
Currently, the duration of the main psychiatry teaching block varies across the various schools responding to this survey, ranging from 6 to 12 weeks, with a mean of 7.6 weeks. In some schools, this time is used exclusively for psychiatry but in others the study of related subjects such as psychology and neurology are also taught within the block.

Although educationalists regard lectures to large groups of students as being a far from optimal method of teaching, such lectures continue to be widely used because they are recognised as being less demanding than the alternatives, both in terms of organisation and resources. Currently, the duration of lectures to large groups in medical schools ranges between 3 and 50 hours, with a mean of 22 hours for each group of students studying psychiatry. More than $90 \%$ of undergraduate medical schools are still reliant on this method of teaching. However, in addition, all medical schools also employ small tutorial groups to teach students, for a range of 3-30 hours, with a mean of 16 hours. The use of a problem-based approach to the teaching of psychiatry appears to be increasing. It is currently employed to some degree in seven medical schools and to a large extent in four schools. It should be noted that such an approach is not necessarily the same as using structured problem-based learning methods. Additional respondents reported that moves towards this teaching method were currently being developed within their medical schools and greater use of this strategy will require more use of small group teaching.

\section{Clinical placements}

The activities engaged in by students while they are attached to psychiatry varies. Most psychiatry material continues to be taught by psychiatrists. There is, however, some increase in the placement of students with related specialists, such as members of community mental health teams. Other possibilities being explored in some schools include the teaching of psychiatry within the setting of general practice, by psychiatrists as well as by general practitioners and community psychiatric nurses.

\section{Assessment}

The GMC recently issued draft guidance on the assessment of medical students. As with the content 
of a core curriculum, the GMC have not, for the purposes of assessment, made any specific recommendations but have simply offered guidelines. It remains up to individual schools to develop assessment methods and link them to teaching, with the important proviso that development of the core curriculum should not be purely assessment-led. The aspects of education that need to be assessed are:

- knowledge and its application

- skills

- attitudes.

Of these, knowledge is the easiest to test and attitudes the most difficult. Schools employ a wide range of assessment methods in their approaches to meeting these goals for psychiatry. The majority (90\%) record some form of end-of-attachment examination in psychiatry. In addition, approximately half the schools have an end-of-year examination in psychiatry. In some, this forms part of the final assessment, but $20 \%$ report that psychiatry does not form part of final medical examinations at the end of the undergraduate course.

A wide variety of assessment techniques are used. All but two schools use some form of continuous assessment, although the precise nature of this varies, from the use of a structured logbook to a general mark given by a consultant to a student who has been attached to a particular clinical placement. The most widely used examination techniques continue to be multiple choice questionnaires and vivas, both reported to be employed in $70 \%$ of schools. In addition, $60 \%$ of schools now also employ objective structured clinical examinations (OSCEs). A smaller number of schools employ short answer questions, essay questions and case commentaries. Although not mentioned in the survey, the employment of video-recordings may also be of value in assessment. Possible uses include asking students to comment on recordings of patients and making recordings of student-patient interactions, which both students and teachers can subsequently comment upon.

All schools report that they examine skills but several respondents note that this is relatively difficult. In general, examination of skills is made either by observed clinical practice, particularly during a patient interview, or in the setting of an OSCE.

There does not, at the moment, appear to be any general consensus about how attitudes should be assessed in psychiatry, or even which particular attitudes should be the focus of attention - those towards mental illness in general, those towards patients with mental illness or even those towards psychiatrists. However, it is of interest to note the results of one recent study of the effect on medical student's attitudes of changing the curriculum. Singh et al (1988), using attitudinal questionnaires briefly covering all three of the above domains, observed that shortening the psychiatric attachment and introducing a problem-oriented approach to replace lectures did not significantly change the students' generally positive attitudes.

\section{Conclusions}

Most of those qualifying as doctors will not receive any further formal education in psychiatry, and yet doctors in almost all areas of practice may come across patients with significant mental health problems. It is, therefore, important to avoid the danger of diminishing undergraduate psychiatry to a point where it does not equip non-psychiatrists for their future careers. It is generally agreed that the immediate aims of undergraduate teaching of psychiatry include the introduction of students to psychiatric phenomenology and ensuring that all qualifying doctors are able to perform a competent mental state examination, with the ability to diagnose the major psychiatric disorders. Despite the time pressures within the curriculum, some space should be found to encourage students to develop the ability to think about normal and abnormal functioning of individuals in the context of their whole life - informed by concepts of biological, psychological and social vulnerabilities, together with the role of provoking, precipitating and perpetuating factors. They should also gain an awareness of how to manage the common psychiatric states that present in, and are often managed within, the primary care setting.

Considering the future of undergraduate education in psychiatry, it is possible that movement towards community-based teaching of psychiatry will increase in importance as medical schools are required to devolve more of their teaching beyond the perimeter of the university hospital, and as these non-hospital clinical structures become more established in psychiatry. However, from the point of view of university-linked teaching departments, the devolvement of teaching to community settings, at least in England, will bring financial implications in terms of alterations in the service increment for teaching.

With respect to the overall structure of the curriculum, psychiatry in some schools is taught in relative isolation, normally towards the end of the clinical course, while elsewhere there is a greater degree of vertical integration, with introduction to some aspects of psychiatry earlier in the course. It 
has been reported that an undergraduate psychiatry curriculum which is fully integrated through the five-year medical course brings advantages over a more isolated style of psychiatry teaching (Carr et al, 1996).

Undergraduate medical education in general, and teaching in psychiatry in particular, are going through a period of major change. This will involve a reduction in the bulk of knowledge that will be communicated to students, and there are pressures to employ teaching methods that move beyond either large lectures or small clinical apprenticeships attached to consultant firms within hospitals. The identification of a core curriculum in psychiatry may result in some of the material that is currently taught no longer being known to the majority of qualifying pre-registration house officers.

The role of apprenticeship in learning how to be a doctor remains something of a controversial issue. Although there may be alternative approaches to acquiring knowledge, in terms of learning clinical skills and attitudes, no convincing alternatives to apprenticeship have yet been proven. There will be a reduction in the time that will be available for core clinical teaching in order to make time for SSMs, but, although it may be possible to support the claim that students currently suffer from factual overload, it is more difficult to support the notion that reducing the time spent in acquiring core clinical skills will not be significant. It will be important in the future that the construction and supervision of SSMs and the nature of individual student SSM portfolios pay attention to learning how to be a doctor as well as to the knowledge base supporting this role-not only in psychiatry but throughout the undergraduate curriculum.

\section{References}

Carr, V. J., Hazell, P. L. \& Williamson, M. (1996) Teaching psychiatry in an integrated medical curriculum. Australian and New Zealand Journal of Psychiatry, 30, 210-219.

General Medical Council (1993) Tomorrow's Doctors: Recommendations on Undergraduate Medical Education. London: GMC.

Singh, S. P., Baxter, H., Standen, P., et al (1998) Changing the attitudes of 'tomorrow's doctors' towards mental illness and psychiatry: a comparison of two teaching methods. Medical Education, 32, 115-120.

Walton, H. \& Gelder, M. (1999) Core curriculum in psychiatry for medical students. Medical Education, 33, 204-211.

Working Party of the Education Committee of the Royal College of Psychiatrists (1997) Core psychiatry for tomorrow's doctors. Psychiatric Bulletin, 21, 522-524.

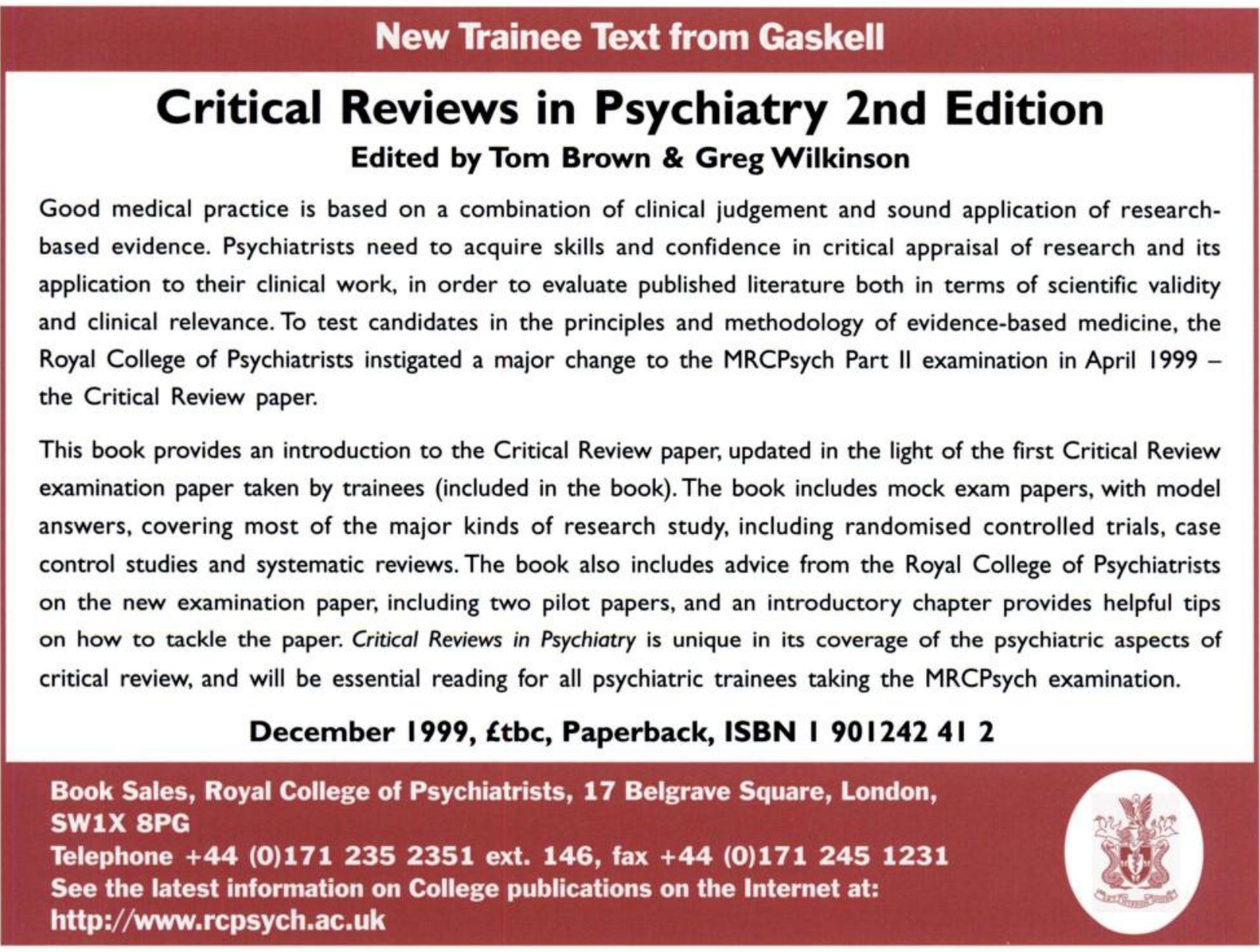

